\title{
COMMENTARY
}

\section{Study shows patients have a positive perception of testosterone nasal gel}

\author{
Thomas De Los Reyes, MD, FRCSC; Dean S. Elterman, MD, MSc, FRCSC
}

Division of Urology, Department of Surgery, University Health Network, University of Toronto, Toronto, ON Canada

Cite as: Can Urol Assoc J 2019;13(12):390. http://dx.doi.org/10.5489/cuaj.6340

See related article on page 384

U rologists and patients may have preconceived notions when it comes to acceptance and usability of various replacement testosterone therapies (TTh). We make assumptions that men will prefer a needle over a patch or a topical gel over a nasal gel. When Natesto ${ }^{\circledR}$ testosterone nasal gel (TNG) was introduced in Canada, we all weren't too sure how it would be accepted. We presumed that nasal administration and twice daily (bid) to three times daily (tid) dosing would either be a big deal, leading to resistance by patients or no big deal, leading to widespread adoption. The truth is, beyond anecdote, we haven't really examined patient preference of TNG vs. topical TTh. The My-T study in this month's issue of CUAJ sheds some light on this subject in both novice and experienced users of replacement TTh.

Lee at al report on patient perceptions with TNG relative to topical TTh. ${ }^{1}$ This is part of a Canadian multi-institution study that recruited hypogonadal males that were either naive or experienced in testosterone therapy, with the majority of patients being the latter. This was a single-arm intervention study with patients treated with TNG for up to 120 days with potential dose adjustment at day 90. Patient satisfaction was assessed using the validated Treatment Satisfaction Questionnaire for Medication, whose questions revolve around effectiveness, convenience, and global satisfaction.
In general, the results of the study showed that patients perceived therapy with TNG to be more effective and convenient compared to baseline regardless of dosing schedule (bid or tid) or whether they were treated with prior testosterone formulations or not. Global satisfaction, in large, was unchanged from baseline.

What is interesting in this study is that patients, the majority of whom were prior testosterone users (experienced), perceived TNG to be more effective and convenient despite the more frequent dosing schedule than the available alternatives. Patient compliance to treatment is an issue that plagues clinical care. The concern that bid or tid dosing will lead to patient dissatisfaction was not demonstrated in this study. In fact, this study sheds positive light on TNG from the practical viewpoint of the patient. It would be interesting to see if this translates into a change in prescribing patterns by both urologists and primary care providers at large.

Competing interests: Dr. Elterman has been a consultant and advisory board member for Acerus. Dr. De Los Reyes reports no competing personal or financial interests related to this work.

\section{Reference}

1. Lee J, Brock G, Barkin J, et al. The My-T study: Patient satisfaction and preference comparing topical and nasal testosterone therapies. Can Urol Assoc J 2019;13:384-9. http://dx.doi.org/10.5489/cuaj.5680

Correspondence: Dr. Dean S. Elterman, Division of Urology, Department of Surgery, University Health Network, University of Toronto, Toronto, ON Canada; Dean.Elterman@uhn.ca 